\title{
Myocardial Involvement in the Marfan Syndrome
}

\author{
Yoshiki Fujiseki, M.D., Keiko Okuno, M.D., \\ Masaki Tanaka, M.D., Morimi Shimada, M.D., \\ Masashi Takahashi, M.D.,* and Katsuyuki Kawanishi, M.D.*
}

\begin{abstract}
SUMmary
A patient with the Marfan syndrome and echocardiographic and angiocardiographic evidence of hypertrophic cardiomyopathy is presented. Endomyocardial biopsy was performed. Histologic abnormalities of the endomyocardium noted in this patient were considered to be related to the basic generalized connective tissue abnormality, and the patient subsequently developed myocardial disease compatible with hypertrophic non-obstructive cardiomyopathy. We believe that this case emphasizes the possible coexistance of subclinical myocardial disease in patients with the Marfan syndrome.
\end{abstract}

\section{Additional Indexing Words:}

Hypertrophic cardiomyopathy Myocardial ultrastructure The Marfan syndrome Collagen fiber Mitral valve prolapse syndrome

THE Marfan syndrome is an abiotrophy with progressive changes involv1 ing many systems, including the skeletal, occular, pulmonary and cardiovascular systems. Cardiovascular abnormalities are found even in infants and small children. ${ }^{1,2}$ Life expectancy is shortened chiefly because of the cardiovascular lesions. ${ }^{3)}$ The major life-threatening complication results from degenerative changes in the media of the aorta due to loss of elastic tissue and accumulation of acid mucopolysaccharides, resulting in aneurysmal dilatation of the aorta, sinuses of Valsalva and the aortic annulus. Mitral regurgitation involving dilatation of the annulus, distorted leaflets, or abnormal chordae tendineae is also frequent.

The endomyocardial lesion in this disease has not been fully discussed. Some investigators noted myocardial fibrosis and hypertrophy in some

From the Departments of Pediatrics and Radiology,* Shiga University of Medical Science, Ohtsu, Shiga, Japan.

Address for reprint: Yoshiki Fujiseki, M.D., Department of Pediatrics, Shiga University of Medical Science, Setatsukinowa, Ohtsu, Shiga 520-21, Japan.

Received for publication September 17, 1984.

Manuscript revised January 14, 1985. 
autopsied cases with the Marfan syndrome. ${ }^{4)-8)}$ We describe a 9 year-old girl with the Marfan syndrome who developed echocardiographic evidence of hypertrophic cardiomyopathy without a pressure gradient. Endomyocardial biopsy revealed myocardial degeneration, accumulation of interstitial ground substance which was considered to be acid mucopolysaccharide, and degenerative collagen fibers which appeared to be fragmented and in disarray. These changes are possibly related to the underlying Marfan syndrome.

\section{Case Report}

A 9 year-old Japanese girl with arachnodactyly was referred to our clinic for evaluation of her cardiovascular abnormalities. A diagnosis of the Marfan syndrome had been made by her ophthalmologist 2 years earlier when she observed bilaterally dislocated lenses. The patient's mother was afflicted with the same disease.

The patient exhibited the characteristic marfanoid habitus. She was at the 50 th percentile for weight $(22 \mathrm{Kg}$ ) and the 90th percentile for height $(125 \mathrm{~cm})$. The extremities were extremely long and slender, particularly the hands and feet. The hand-height ratio was greater than $11 \%$ and the foot-height ratio greater than $15 \%$. The palate was high and arch shaped. There was ichtyosis vulgaris of the extremities. The lungs were clear and the liver was not enlarged. Apical auscultation revealed a midsystolic click and a late systolic murmur with a third protodiastolic sound. The second heart sound was normally split and the pulmonary component was of normal

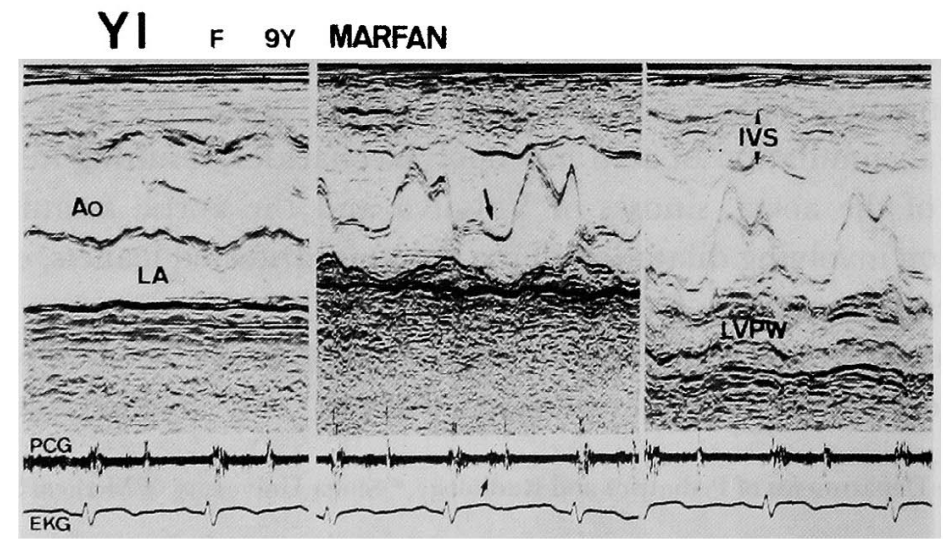

Fig. 1. M-mode echocardiogram. Mitral valve prolapse of the anterior mitral leaflet (large arrow) and disproportionate septal thickening are observed (IVS: $18 \mathrm{~mm}$, LVPW: $7 \mathrm{~mm}$ ). 
intensity.

The electrocardiogram showed non-specific ST-T changes in II, III, $\mathrm{aV}_{\mathrm{F}}, \mathrm{V}_{5}$ and $\mathrm{V}_{6}$. Chest roentgenogram was not contributory. M-mode echocardiogram showed dilatation of the aortic root, late systolic buckling of the mitral valve and asymmetric septal hypertrophy (IVS/LVPW $=2.5$ ) (Fig. 1) without anterior movement of the mitral valve during systole. A two-dimensional echocardiogram disclosed that the anterior mitral valve was prolapsed into the left atrium during late systole and the interventricular septum was disproportionately thickened.

Right and left heart catheterization studies were performed with the left ventricle entered in retrograde fashion. No pressure gradient was noted in the left ventricle. There was no elevation of the end-diastolic pressures in either the left or right ventricle. But a left ventriculogram in the right anterior oblique position showed marked deformity of the left ventricular chamber with mild obstruction of the mid-ventricle and abnormal bulging of the mitral valve into the left atrium during late systole. Moderate mitral regurgitation was also present in late systole (Fig. 2). Catheterization data are summarized in Table I.
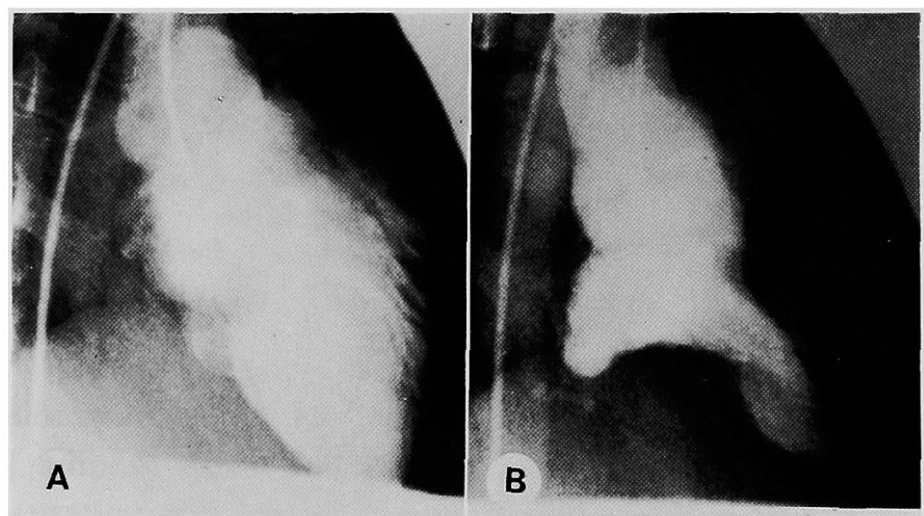

Fig. 2. Left ventriculogram. A: end-diastole, B: end-systole. Moderate thickening of the left ventricular free wall in end-diastole and mild midventricular obstruction in end-systole are noted.

Table I. Cardiac Catheterization Data

\begin{tabular}{|c|c|c|c|}
\hline I & Pressure $(\mathrm{mmHg})$ & \multicolumn{2}{|c|}{ II. Volume } \\
\hline RA & $\mathrm{a}=3, \mathrm{v}=1, \mathrm{~m}=1$ & LVEDV & $54.1 \mathrm{ml}$ \\
\hline $\mathrm{mPA}$ & $25 / 10, m=17$ & LVESV & $4.9 \mathrm{ml}$ \\
\hline LPG & $\mathrm{m}=6$ & $\mathrm{EF}$ & $91 \%$ \\
\hline $\mathrm{AO}$ & $105 / 70, m=84$ & LVmass & $98.6 \mathrm{~g}$ \\
\hline $\mathrm{LV}$ & $100, E D P=7$ & & \\
\hline
\end{tabular}




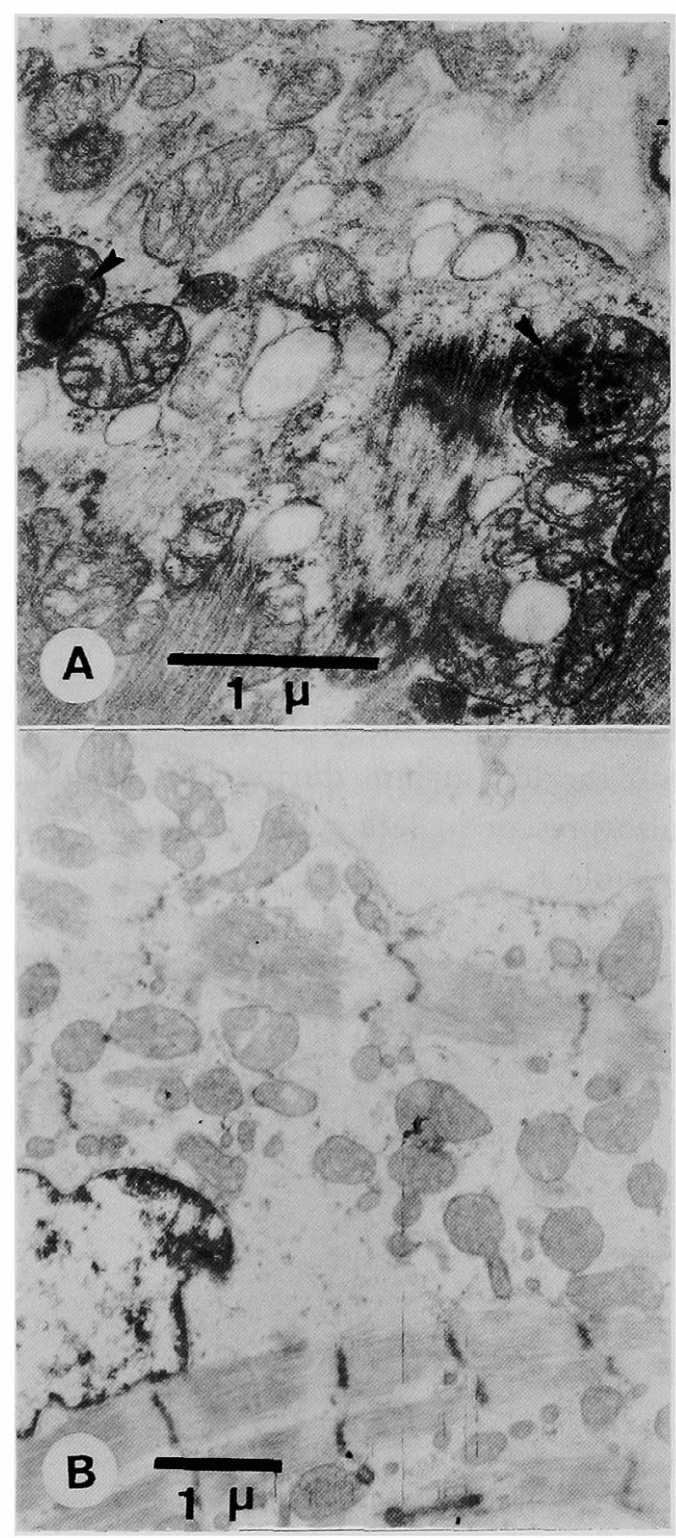

Fig. 3. Electron microscopic findings of the myocardiocytes. Disruption of myofibrils and myofibrillar lysis can be observed (B). Some mitochondria contain small irregular dense bodies (arrow head, A).

His bundle electrogram revealed a normal A-H interval $(72 \mathrm{msec})$ and a prolonged $\mathrm{H}-\mathrm{V}$ interval $(70 \mathrm{msec})$.

After routine cardiac catheterization, an endomyocardial biopsy was performed using a long sheath and flexible bioptome. Multiple right ventricular endomyocardial specimens were procurred from the interventricular septum. 


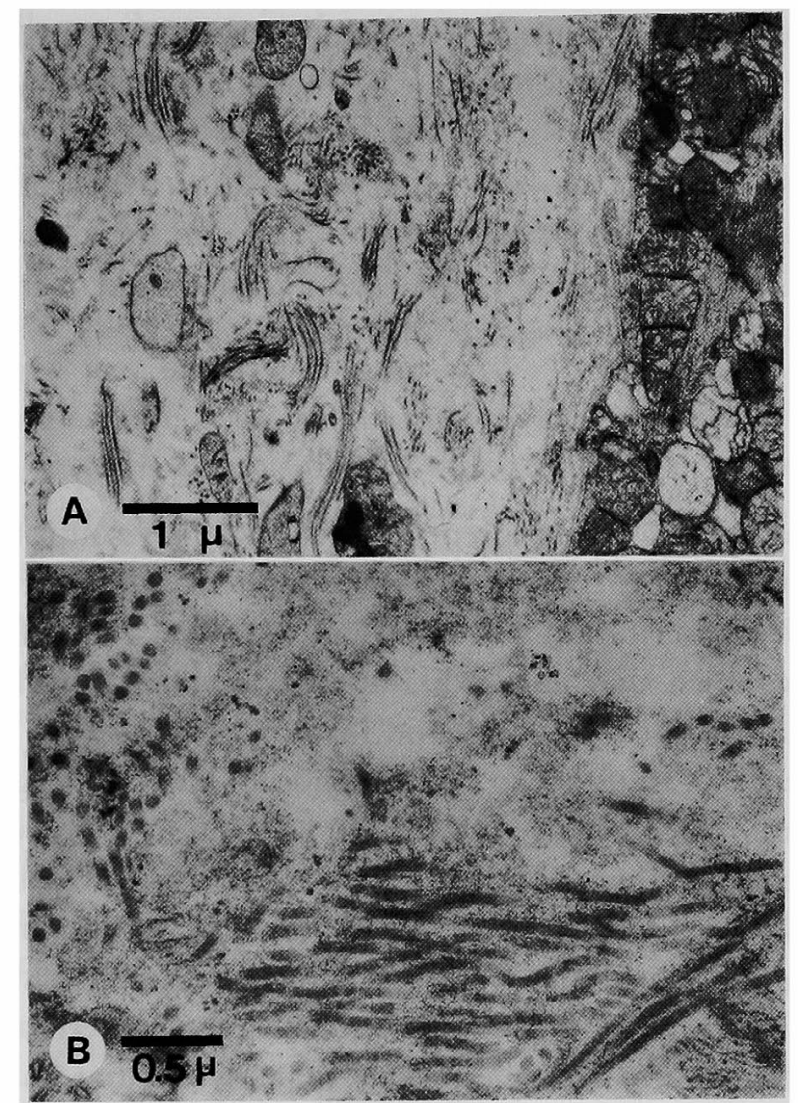

Fig. 4. Electron microscopic findings of the interstitium. Collagen bundles are unravelled and loosely organized $(A, \times 12,000)$. Collagen fibers show an apparent loss of typical periodic banding pattern $(B, \times 30,000)$.

Under light microscopy, the endomyocardial biopsy tissue revealed a normal endocardium and no hypertrophied myocardiocytes. Focal endomyocardial adipose tissue and mild myocardial fiber disarray were noted. The fragmentation and loss of myocardial fibers with interstitial edema were also noted in some areas of the biopsied specimens. Interstitial fibrosis was demonstrated by Masson's trichrome stain.

Under electron microscopy, the most prominent ultrastructural feature concerning the myocardiocytes was the disruption of the myofibrils and myofibrillar lysis. Some mitochondria contained small, irregular, dense bodies with swollen and disrupted cristae (Fig. 3A). Lipid droplets were also noted in the mildly degenerated myocardiocytes. Mild proliferation of the fibroblast was observed in the vicinity of the degenerated cells, but large areas showed an apparent loss of collagen tissue, with the individual fibrils fragmented, coiled and disrupted. A few particles, nearly equal in size to the 
fibrils, were often noticed around or intimately associated with the fragments of fibrils. The most obvious change in the intrinsic structure of collagen fibrils in the myocardial interstitium was an apparent loss of typical periodicity in the transverse bands (Fig. 4B). There was no demonstrable pool of acid mucopolysaccharide, but increased amount of ground substance in areas where destruction of collagen tissue was present. Elastic stain using tannic acid ${ }^{9 !}$ disclosed decreased elastic fibers in the stroma.

\section{Discussion}

Cardiovascular lesions in patients with the Marfan syndrome are the main cause of the poor life expectancy. The prominent anomalies include aortic dilatation, aortic regurgitation and mitral valve prolapse. Echocardiographic observation has shown that aortic dilatation and silent mitral valve prolapse are already manifest early in childhood in most patients. ${ }^{2}$

The principal pathology in the ascending aorta is fragmentation and sparsity of elastic fibers, irregular whorls of seemingly hypertrophied, and perhaps hyperplastic, smooth muscle cells, an increase in collagenous tissue, a marked increase in the vascularity of the tunica adventitia and tunica media and the presence of cystic spaces occupied by material that stains metachromatically. ${ }^{9)}$ The aortic and valvular lesions are so severe and conspicious in contrast with the heart muscle lesions that myocardial changes were not fully studied in the past. A few papers reported that myocardial hypertrophy and fibrosis were observed in selected autopsied cases with the Marfan syndrome. ${ }^{4-8)}$ It remains uncertain whether the myocardium was affected by the basic metabolic process in this disease or that the myocardial alterations were secondary to the valvular heart lesion of this disease. To our knowledge, there are thus far no reports on the coexistance of the Marfan syndrome and hypertrophic cardiomyopathy.

Endomyocardial biopsy in our case revealed that myocardiocytes were not hypertrophied but degenerated and that there was mild proliferation of fibroblasts with apparent loss of the elastic fibers and degenerative collagen fibers. There were no demonstrable pools of ground material, but the loosely textured matrix with a few unravelled collagen fibers was increased. These findings suggest that an intimate relationship exists between the myocardial lesions and the basic metabolic abnormality in the Marfan syndrome. A disease process similar to the cystic medial necrosis of the aorta may occur in the myocardium, thus causing an alteration in connective tissue and myocardial degeneration.

Renteria and his colleagues ${ }^{10,11)}$ noted that a granular cell containing 
intracellular collagen deposits in the atrioventricular valve was a characteristic finding in patients not only with Hurler's syndrome but also with mitral valve prolapse syndrome. However, we could not find such cells in the endomyocardial biopsy specimen of this patient.

The extensively woven collagen explains the increased stiffness of the heart. The collagen struts connecting adjacent myocardiocytes would prevent any slippage either in a transverse or lateral direction. ${ }^{12), 13)}$ On the other hand, the collagen was found to be more soluble in several cases of the Marfan syndrome. Furthermore, a defect in the packing of collagen molecules into fibrils was suggested by our finding of an intrafibrillar abnormality. Since abnormal fibrils are generally degraded more rapidly than normal ones, degenerative changes in collagen may occur, thus leading to disruption of the myocardial structure. Degenerative collagen tissue and myocardium resulted in the contraction abnormality seen on angiocardiography. The loose connective tissue in the mitral valve also resulted in mitral valve prolapse. We postulate that the myocardial disease was essentially secondary to the defective organization of collagen into fibrils and the accumulation of acid mucopolysaccharide in the myocardial stroma.

We believe that this case emphasizes the possible coexistence of subclinical myocardial disease in patients with the Marfan syndrome.

\section{ReFERENCES}

1. Phornphutkul C, Rosethal A, Nadas AS: Cardiac manifestations of Marfan syndrome in infancy and childhood. Circulation 47:587, 1973

2. Sisk HE, Zahka KG, Pyeritz RE: The Marfan syndrome in early childhood, Am J Cardiol 52: 353,1983

3. Murdoch JL, Walker BA, Halpen BL et al: Life expectancy and causes of death in the Marfan syndrome. New Engl J Med 286: 804, 1972

4. Tobin JR, Bay EB, Humphrey EM: Marfan's syndrome in the adult. Arch Intern Med 80: 457,1947

5. Marvel RJ, Genovese PD: Cardiovascular disease in Marfan's syndrome. Am Heart J 42: 814,1951

6. Whitfield AGW, Arnott WM, Stafford JW: "Myocarditis" and aortic hypoplasia in arachnodactyly. Lancet 1: 1387, 1951

7. Van Buchem ESP: Arachnodactyl heart. Circulation 20: 88, 1959

8. Raghib G, Jue KL, Anderson RG et al: Marfan's syndrome with mitral insufficiency. Am J Cardiol 16: 127, 1965

9. Roberts WC, Honig HS: The spectrum of cardiovascular disease in the Marfan syndrome. Am Heart J 104: 115, 1982

10. Renteria VG, Ferrans VJ: Intercellular collagen fibrils in cardiac valves of patients with the Hurler syndrome. Lab Invest 34: 263, 1976

11. Renteria VG, Ferrans VJ, Jones $\mathrm{M}$ et al: Intracellular collagen fibrils in prolapsed ("Floppy") human atrioventricular valves. Lab Invest 35: 439, 1976

12. Caulfield JB, Borg TK: The collagen network of the heart. Lab Invest 40: 364, 1981 
13. Borg TK, Ranson WF, Moslehy FA et al: Structural basis of ventricular stiffness. Lab Invest 44: 49, 1981

14. Scheck M, Siegel RC, Parker J et al: Aortic aneurysm in Marfan's syndrome. J Anat 219 : 645,1979

15. Byers PH, Siegel RC, Peterson KE et al: Marfan syndrome. Proc Nati Acad Sci 78: 7745, 1981 